
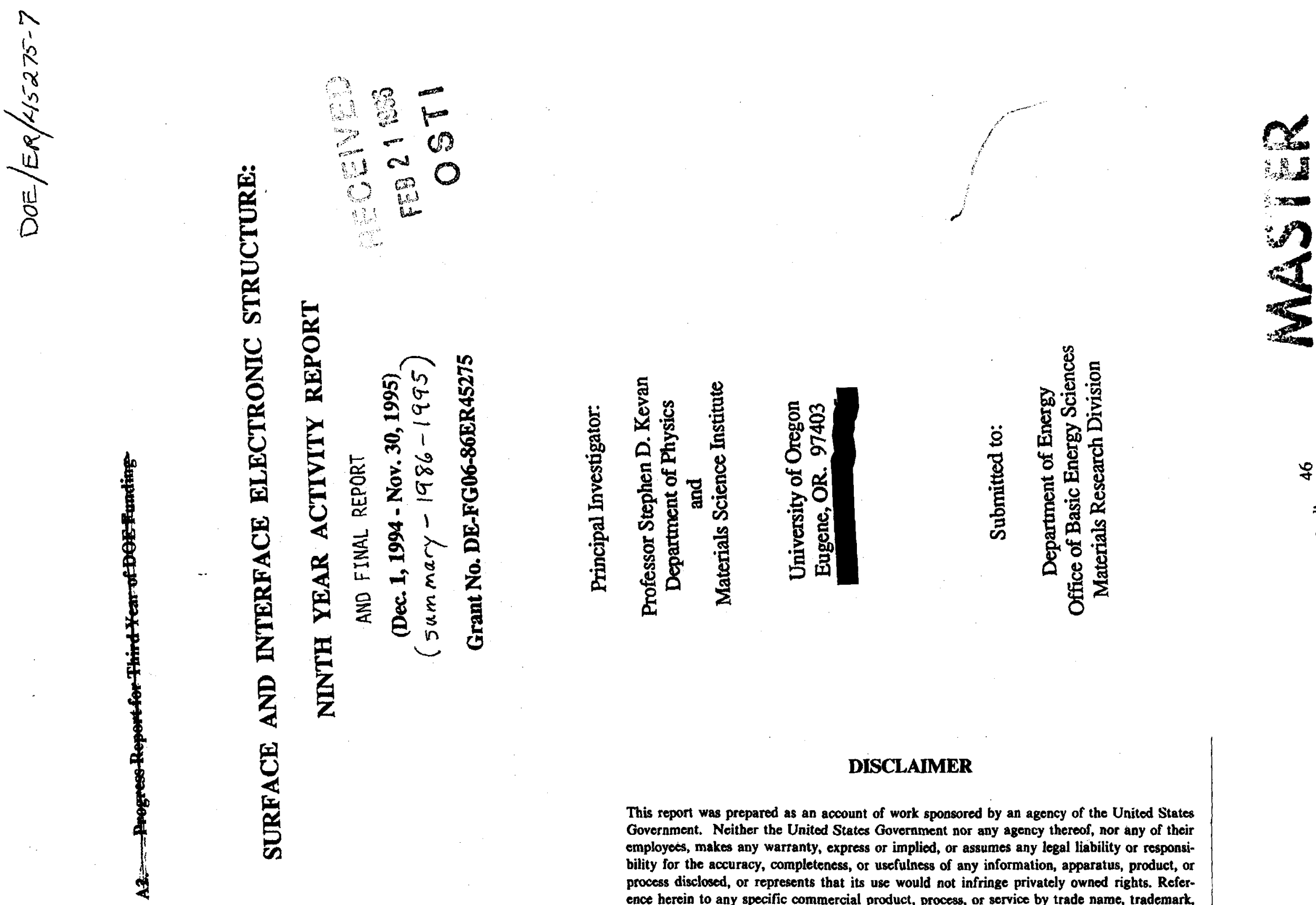

DISCLAIMER

This report was prepared as an account of work sponsored by an agency of the United States Government. Neither the United States Government nor any agency thereof, nor any of their employees, makes any warranty, express or implied, or assumes any legal liability or responsibility for the accuracy, completeness, or usefulness of any information, apparatus, product, or process disclosed, or represents that its use would not infringe privately owned rights. Reference herein to any specific commercial product, process, of service by trade name, trademart, ot necessarily constitute on or United States Government of any agency thereof. The views and opinions of authors expressed herein do not necessarily state or reflect those of the United States Government or any agency thereof. 


\section{PROGRESS REPORT: SURFACE AND INTERFACE ELECTRONIC STRUCTURE}

A year an a half ago, we terminated our operations at the National Synchrotron Light Source and moved our research program to the Advanced Light Source in Berkeley. This move was motivated primarily by the exciting possibilities and research directions made possible by operation at the newer ALS facility. The ALS facility was commissioned in October, 1993. We are members of a 'Participating Research Team' (PRT) associated with undulator beamline 7.0 at the ALS. This beamline was commissioned for experiments the following winter and spring of 1994 . We have thus had about a year to debug the beamline and to try to get some experiments accomplished. Reportedly due to insufficient operating funds, the facility provided beam to users only nine 8-hours shifts per week. In the past year, our program has been devoted primarily to becoming operational at the ALS and to assembling experiments and experimental equipment associated with our program there.

The postdoc currently supported by this grant (Eli Rotenberg) has been stationed at the ALS since the facility was commissioned. Along with Jonathon Denlinger, also supported by a DOE grant to Brian Tonner at the University of Wisconsin, he has been one of the two postdocs primarily responsible for commissioning the beamline and core level photoemission system. Happily, the two of them have made rapid progress in these endeavors and the future is indeed very bright.

Since we are active members of the PRT with personnel on site, we tend to get involved in three generic types of experiments. Firstly, we have some ideas of our own to pursue more or less independently of anyone else in the PRT. The time for these has been and continues to be very scarce since many groups are involved and the demand for time is high. The bulk of our activities in the past year have involved the other two kinds of experiments. There are firstly those in which we are "major collaborators', meaning we contribute with other groups to all phases of the experiment, from conception through analysis and publication. Finally, there are experiments in which we play a more supporting role to help other groups with their experiments. This last type of experiment can be very enjoyable and can even take more time and effort on our part than the first two. Before describing a few initial results from the first two categories, we briefly review those from the third:

- We have helped install a soft x-ray emission spectrograph belonging to Joseph Nordgren from Uppsala University in Sweden, and have participated in a small number of the many experiments his group has accomlished in the past year.

- We have collaborated with Brian Tonner's research group to collect resonant photoemission spectra from thin films of $\mathrm{C}_{60}$ and polystyrene. These are among the first RESPES studies above the $\mathrm{C} \mathrm{K}$ edge, and have lead to a new model for the relaxation processes near a core hole in these systems. 
- We have collaborated with Norm Edelstein and David Shuh of LBL to measure the core level photoemission spectra from nano-Curie quantities for transuranic elements. This was an amusing experiment, since safety personnel outnumbered researchers by a significant margin. We got some good press for our efforts - it was written up in the DOE's ER Newsletter a few months back. A proposed application in verification of compliance to non-proliferation treaties seems far in the future.

We have made progress in three distinct areas described in the following sections.

\section{A. Core Level Photoemission and XPD}

The first functional end station on beamline 7.0 was optimized to perform core level photoemission. Most of our accomplishments in the first year have been in this area, and essentially all of these have been in collaboration with Brian Tonner and his postdoc Jonathan Denlinger. This system is able to accumulate a photoemission spectrum with excellent statistics and resolution in typically less than a minute. The apparatus has been mechanized to an extent that a set of complete, normalized spectra can be accumulated over a large range of emission angles under computer control. These capabilities provide one of the favored methodologies for accumulating $x$-ray photoelectron diffraction (XPD) data and, related to this, something that has come to be called a photoelectron hologram. Photoelectron diffraction is now a mature field. 1-3 Indeed, the P.I., in his PhD thesis, laid some of the groundwork for this technique, although he has not practiced it much over the intervening period. As its name implies, XPD invloves using the interference between the directly photoemitted and substrate back-scattered emission paths to probe surface geometric structures. If a large enough data set is available, then these diffraction patterns become spherical holograms that can be directly inverted to provide a 3D image in the vicinity of the emitting atom.

Over the past year, we have been involved in four separate XPD experiments:

- As a tune-up, we have accumulated a large '3D' data set - a large array of angles collected at many photon energies - on clean $\mathrm{Cu}(001)$. This can be inverted using standard techniques to yield the known structure of copper.

- One of the real strengths of XPD over most other surface structural techniques is simultaneous access to chemical and structural information. This is done by utilizing high energy resolution so that chemically shifted core levels can be resolved and their XPD patterns or holograms measured independently. The first such experiments we have undertaken have been on $\mathrm{Si}(111)-(7 \times 7)$ and Si(001) - (2x1). Both of these structures are fairly well understood, so this can also be considered a tune-up experiment to a degree. However, there is substantial discord in existing assignments of the observed shifted core levels to particular surface atoms in the reconstruction. There is thus significant 
interest in these experiments since the the origin of surface core level shifts and their relation to structure remains an active field, particularly on semiconductor surfaces.

- We have a partial XPD data set for the surface core levels on W(011). Again, our experiment is a test of a fairly well-known structure, though it is by no means a trivial effort given the reactivity of this surface. After we have completed the clean surface, we will undertake the hydrogen-covered surface which has been shown to undergo a (1x1) reconstruction in which the surface layer shifts laterally across the second layer. 4

- Finally, we have started and nearly completed an experiment on ultrathin manganese films on $\mathrm{Cu}(001)$. This prorotypical thin film magnetic metal system has an unknown structure and provides an opportunity to correlate magnetic and geometric structures.

\section{B. Coherent Soft X-ray Scattering}

Substantial interest exists in using the high brightness of second and third generation synchrotron radiation facilities to produce and use spatially coherent $x$-ray and soft $x$-ray beams for a number of applications. One of the P.I.'s primary motivations for moving to the ALS was the possibility of being involved in the qualitatively new science offered by this possibility. The particular research direction we have chosen to pursue in this regard is the scattering of coherent soft $x$-rays off fluctuating systems. Our motivations for pursuing such experiments will be discussed in more detail in the accompanying renewal proposal. Here, we will describe our first efforts to overcome the technical obstacles involved.

A spatially coherent light beam is one with wavefronts that are well-defined transverse to the propagation direction. This is generally the case in light emitted by well-designed lasers, but reasonably intense spatially coherent $x$-ray beams have only recently become avaliable. The high brightness of the ALS will provide substantially more 'coherent soft $x$-ray power' - that is - more photons in a spatially coherent beam - than has been available elsewhere. There are (at least) two ways to produce a spatially coherent beam on an undulator beamline at the ALS. Both rely fundamentally upon the fact the spatial coherence length of a light beam increases upon propagation. The mathematical statement of this is the van Cittert - Zernike theorem from optics, which relates the mutual coherence function of a partially coherent beam to the far-field diffraction pattern of a similarly-defined coherent beam. The two ways to produce spatially coherent soft $x$-rays at the ALS are:

- Up to about $500 \mathrm{eV}$ photon energy, the light emitted in the central cone by the $5 \mathrm{~cm}$ undulator on beamline 7.0 is spatially coherent due to the small emittance of the electron beam inside the undulator. Simply by placing a small (water-cooled) aperture in the undulator beam, one 
automatically gets spatially coherent light. At present, the U7.0 undulator beamline does not provide access to the raw undulator beam. However, a small group of researchers, of which we are members, has been assembled by Malcolm Howells of the Center for X-ray Optics at LBL to establish this capability within the next 6-12 months. This alternative will provide the highest possible flux and excellent spatial coherence with the disadvantage of having only fair temporal coherence $(\lambda \Delta \lambda \sim$ 100). In many applications, the temporal coherence is not particularly important and this addition to the beamline will be very useful to us.

- In principle, the limited temporal coherence of the direct undulator beam can be improved with a 'coherence length stretcher', i.e., a monochromator, without adversely impacting the spatial coherence properties. Indeed, a monochromator is installed on the U7 undulator and is used in essentially all other experiments. Unfortunately, the use of grazing incidence optics in a soft x-ray beamline implies figure errors and short-scale roughness in the optical surfaces that leaves the exit beam with ill-defined spatial coherence properties. The common technique for solving this problem is to insert two small apertures after the exit slit of the monochromator, one near the refocus point and another slightly downstream which is sized to accept the coherent fraction of the beam according to the van Cittert - Zernike theorem.

In either of these two cases, the coherent power scales like the square of the wavelength, and the expected flux of coherent light is $\sim 10^{13}$ and $\sim 10^{9}$ photons/sec in the two cases above for photon energies near the carbon edge $(280 \mathrm{eV})$. In December 1994, in collaboration with Larry Sorensen and Allen Price from the University of Washington, we attempted an experiment along the lines of the second design and achieved a coherent flux within an order of magnitude of the expected level. The power spectrum of the coherent light was extensively tested and was found to be adequate to measure temporal fluctuations on a frequency scale of $1 \mathrm{~Hz}-1 \mathrm{Mhz}$. This actually is a substantial and important result. Considering the mechanical and electronic instabilities that can be present along a 30 meter beamline, by the time one directs the beam exiting the monochromator through two $10-20$ micron apertures separated by $300 \mathrm{~mm}$ to separate the spatially coherent fraction, it is not obvious that the noise spectrum will have low enough amplitude to allow useful measurements of 'real' fluctuating signals. We showed that it does, but a substantial number of problems precluded any real experiments using this coherent light in our December nu. We intend to pursue experiments along these lines again in the near future.

\section{Low Angle Diffraction from Nanostructures}

An increasing fraction of the work on this project involves scattering of $x$-rays and soft $x$-rays from thin films and nanostructured materials. This reflects our movement away from studies of the electronic structure of nominally clean surfaces and toward synthesis and analysis of exotic materials, as 
described more fully in the following renewal proposal. For this reason, in the fall of 1993, we wrote and were awarded a DOE University Research Instrumentation proposal to purchase and modify a new diffractometer. This will be utilized to a substantial degree to supplement our ALS activities. Since the URI program provides exclusively funds for instrumentation, the graduate student supported by this grant is involved in modifying and commissioning this apparatus. We describe this activity briefly here, while a more complete discussion of the science we will pursue is left for the renewal proposal.

The diffractometer purchased is a $\theta-\theta$ diffractometer with sufficient precision that low-angle diffraction from layered materials and thin films with $d$-spacings in the 10-10000 $\AA$ range can be measured. The modifications involve 1) adding analyzing crystals to both arms of the diffractometer so that high resolution can be achieved, 2) allowing for automated sample alignment with adequate precision for meaningful low-angle studies, and 3) addition of a high vacuum environmental chamber so that low and moderately high temperature studies can be undertaken in situ. Surprisingly, such an apparatus is not commercially available despite several obvious applications in thin film technology. Several companies have communicated substantial interest in such an apparatus. This had made the manufacturer (Scintag, Inc.) keenly interested in these modifications, and we are actively collaborating with them on this project. At present, all parts except the vacuum chamber have been completed and are being installed.

\section{References:}

1. W.F. Egelhoff, Crit. Rev. Solid State and Mat. Sci. 16, 213 (1990).

2. C.S. Fadley, in Synchrotron Radiation Research: Advances in Surface Science, R.Z. Bachrach, ed. (Plenum, New York, 1990).

3. D.P. Woodruff, in Angle-Resolved Photoemission, S.D. Kevan, ed. (Elsevier, Amsterdam, 1992).

4. J.W. Chung, S.C. Ying, and P.J. Estrup, Phys. Rev. Lett. 56, 749 (1986). 


\section{PUBLICATIONS AND PRESENTATIONS FROM SUPPORTED WORK}

\section{A. Publications}

\section{Research Articles:}

1. "Delocalization of the Fe $3 d$ Levels in the Quasi-2D Correlated Insulator FePS 3 ", Won-Kook Choi, E. Kneedler, and S.D. Kevan, Phys. Rev. B 50, 15276 (1994).

2. "Fermi Contours and Adsorbate Periodicities: $\mathrm{O} / \mathrm{Mo}(011)$ and $\mathrm{O} / \mathrm{W}(011)^{n}, \mathrm{~S}$. Dhar, K.E. Smith, and S.D. Kevan, Phys. Rev. Lett. 73, 1448 (1994).

3. "Alkali Chemisorption on Ta(011)", E. Kneedler, A.B. Andrews, and W.-K. Choi, and S.D. Kevan, Phys. Rev. B. 51, 1823 (1995).

4. "Low Angle X-ray Diffraction as a Probe of Reactions at Buried Interfaces and as a Characterization Technique for Thin Films", T. Novet, S.D. Kevan, and D.C. Johnson, Mat. Sci. and Eng. XXX, XXXX (1995).

5. "First Results from the Spectromicroscopy Beamline at the Advanced Light Source", J.D. Denlinger, E. Rotenberg, T. Warwick, G. Visser, J. Nordgren, J.H. Guo, P. Skytt, S.D. Kevan, K.S. McCutcheon, D. Shuh, J. Bucher, N. Edelstein, J.G. Tobin, and B.P. Tonner, Rev. Sci. Inst. 66, XXXX (1995).

6. "Surface Core Level Photoelectron Diffraction of Surface Reconstructions", E. Rotenberg, J. Denlinger, S.D. Kevan, and B.P. Tonner, Mat. Res. Soc. Proc. XXX, XXXX (1995).

7. "Surface States on Metals", S.D. Kevan, book chapter in Electronic Structure of Surfaces, K. Horn and M. Scheffler, eds. (Elsevier, Amsterdam, 1995).

Presentations on Sponsored Research:

1. "Are Surface States Important?", U of O Condensed Matter Physics Seminar delivered by S. Kevan, October, 1994.

2. "First Results from the Spectromicroscopy Beamline at the Advanced Light Source", Synchrotron Radiation Instrumentation 1994, talk delivered by J.D. Denlinger.

3. "Surface Core Level Photoelectron Diffraction of Surface Reconstructions", Annual Meeting of the Materials Research Society, Boston, November, 1994, talk delivered by E. Rotenberg.

4. "Core Level Photoelectron Diffraction Studies of Silicon Surface Reconstructions", March Meeting of the American Physical Society, March, 1994, San Jose, talk delivered by E. Rotenberg.

5. "Synchrotron Photoelectron Spectroscopy Studies of Actinides", March Meeting of the American Physical Society, March, 1994, San Jose, talk delivered by J. Denlinger. 


\section{THREE YEAR ACTIVITY REPORT}

\section{A. Angle-Resolved Photoemission}

Since this project's inception, we have focused much of our attention on the electronic structure of clean and adsorbate-modified transition metal surfaces. Our motivation for these studies has been to forge a connection between surface electronic structure and low energy excitations such as surface phonons and adsorbate vibrations. For example, our early studies of the surface Fermi contours on $\mathrm{W}(011)^{1}$ and $\mathrm{Mo}(011)^{2}$ were instrumental in helping us to understand the subtle differences in reconstructive behavior and dipole layer formation between these two. Moreover, our predictions of phonon damping motivated detailed measurements of surface phonon dispersion relations. These have provided the best current example of a surface phonon anomaly, ${ }^{3}$ a result which is qualitatively though not quantitatively predicted by our Fermi contours and which has also motivated serious theoretical attention. ${ }^{4}$ Our efforts in this area continued during our final 1-2 years at the NSLS, and we breifly review these results here.

\section{Fermi Contours: $\mathrm{O} / \mathrm{Mo}(011)$ and $\mathrm{W}(011)$}

One of the most commonly observed yet most poorly understood phenomena in modem surface chemical physics concerns the lateral potential energy surfaces which conspire to produce the variety of observed superlattice periodicities in adsorbed layers. The source of this difficulty is the substrate itself since this mediates the lateral interactions in a complex way. While useful empirical models for substratemediated interactions have existed for over 20 years, $5-14$ a precise, nearly first principles characterization of these on an energy scale relevant to most surface processes has not been available. ${ }^{15}$ One of the empirical models, which is relevant to metal surfaces, utilizes the existence of well-defined Fermi contours. These support Friedel-like oscillations that can lead to an oscillatory lateral interaction potential. 16 We have recently measured surface Fermi contours for ordered oxygen layers on Mo(011) and W(011), and shown that they provide a mechanism to explain some of the remarkable qualitative differences observed in the low-coverage phase diagrams for these systems. 17

Collection of many ARP spectra throughout the surface Brillouin zone (SBZ) allows direct determination of 2D Fermi contours. As shown in Fig. 1, at 0.25 monolayer coverage of oxygen on Mo(011), two hole pockets exist, one along each edge of the SBZ. These are are nearly the same size and shape, although there is no reason by symmetry why this needs to be the case. The hole pockets are centered in the $(2 \times 2)$ superlattice Brillouin zones, shown as dotted lines in Fig. 1. This situation implies what we have called a "global" Peierls distortion, 18 where each point on one of these two contours has a tangent which is parallel to the tangent of a point on the other contour. Each pair of such points is necessarily separated very nearly by a reciprocal lattice vector for the $(2 \times 2)$ net. This provides a logical and purely electronic driving force for producing the observed $(2 \times 2)$ ordering pattern for this system. These contours are also consistent with a weaker, longer range reconstruction of the $(2 \times 2) \mathrm{O} / \mathrm{Mo}(011)$ 


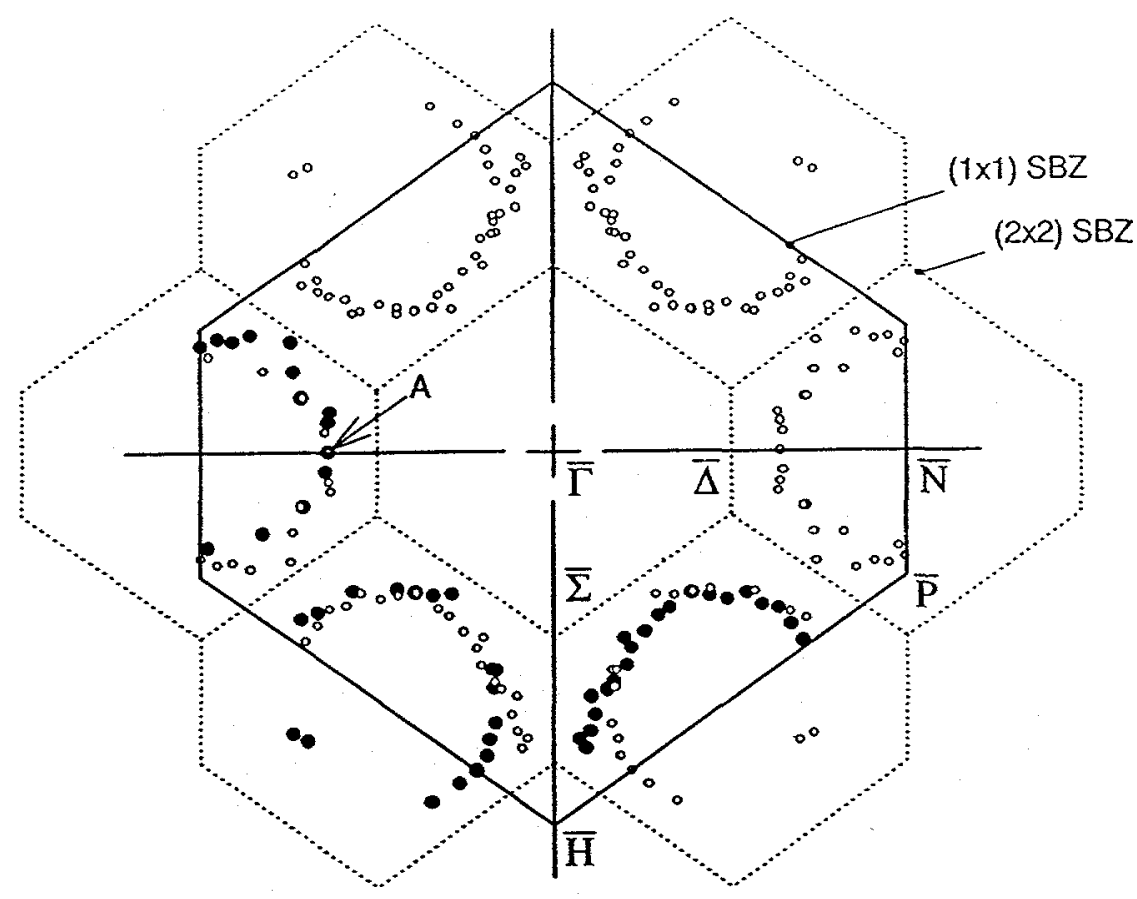

Fig. 1: Fermi Contours for Mo(011)-(2x2)O.

surface through a normal Peierls distortion. ${ }^{19}$ Neither this long-lange reconstruction nor the 0.25 monolayer $(2 \times 2)$ superlattice is observed for $\mathrm{O} / \mathrm{W}(011)$, and none is predicted by our Fermi conoturs for that system.

\section{Alkali Adsorption on Ta(011)}

The chemisorption of alkalis on metals has received much attention for some time, particularly in recent years. ${ }^{20}$ From a practical standpoint, alkalis have long been known to affect surface processes, including catalytic promotion and enhancement of photoreceptivity. More fundamentally, the monovalent alkalis are a relatively simple adatom in ordinarily complicated systems of chemisorption. Perturbation by alkali adsorption will help determine how a known clean surface interacts with its environment. Recently, there has been quite a bit of discussion about the nature of the bonding in alkali adsorption - ionic, covalent, or semantic. ${ }^{21}$ Our approach to this problem has been from the prespective of the substrate's electronic structure, and our results clearly indicate the importance of alkali-ns/metal-d hybridization for alkali adsorption on $\mathrm{Ta}(011) .22$ One might like to call this 'covalent', but given the multiplicity of interpretations that term can adopt we tend to avoid its usage in this context.

In previous studies, we have used the relative simplicity of the $\mathrm{Ta}(110)$ surface electronic structure to provide insight into interactions between a surface layer and the bulk. 23,24 On the clean surface, a single, predominantly $d_{z^{2}}$ surface state of even symmetry exists within a large projected Ta bulk band gap at zone center. Upon hydrogen chemisorption, the state at zone center shifts nearly $2 \mathrm{eV}$ discontinuously to higher binding energy. The pure state at zone center is dispersionless until near the point where it crosses the edge of the bulk band continuum, for both clean and hydrogen-covered 


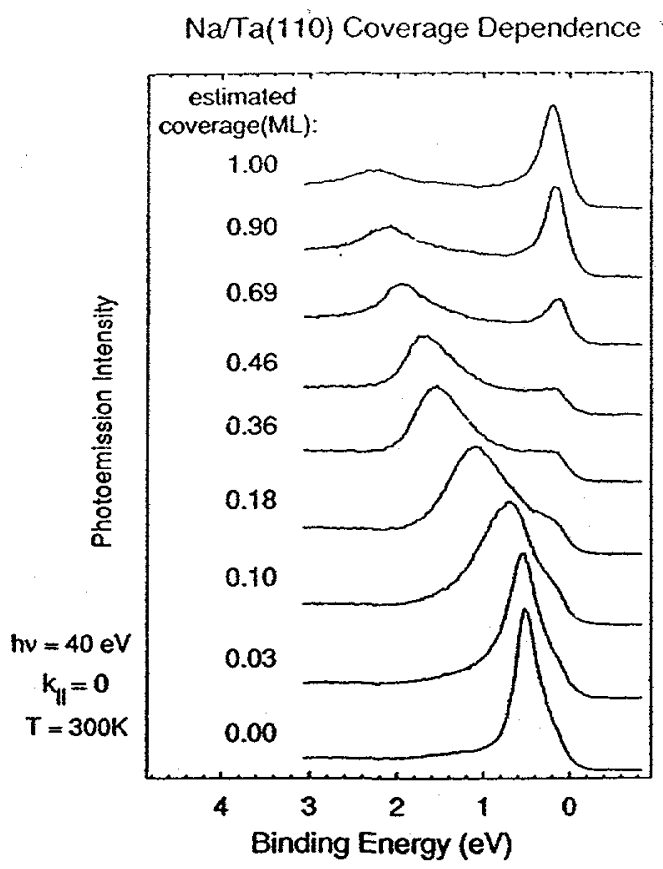

Fig. 2: ARP spectra collected at normal emission for various coverages of $\mathrm{Na}$ on $\mathrm{Ta}(011)$.

surfaces. The lack of dispersion and the discontinuous shift of the hydrogen-modified surface state indicate that it remains laterally localized upon hydrogen adsorption.

We found the interaction of the alkali ns levels with the $\mathrm{Ta}(110)$ surface state to be quite different from this. 22 Fig. 2 shows ARP spectra collected at normal emission for various coverages of $\mathrm{Na}$ on $\mathrm{Ta}(011)$. The clean surface state, seen as a large photoemission peak near the Fermi level $\mathrm{E}_{\mathrm{F}}$ in the bottom spectrum, is continuously rather than discontinuously shifted and is also slowly quenched with increasing coverage. The surface state also acquires substantial dispersion as alkali is adsorbed, with an effective mass approaching the free electron mass at monolayer coverage. These coverage-dependent binding energies and effective masses vary systematically as a function of alkali identity - for heavier alkalis the shifts are smaller and the masses larger. The reduced photoemission intensity, acquired dispersion, and overall energy shift of the alkali-modified surface state suggest that the surface-state orbital character has changed substantially through hybridization with the extrinsic the alkali ns level. We have developed a very simple yet unified model to relate this observed surface state shift to the dramatic reduction of work function as the alkali coverage increases. The model is 'covalent' in nature, since it is based upon simple hybridization integrals. Of course, the model ignores the width of the alkali band at finite coverage which can best be incorporated using CPA-like computational techniques.

\section{Quasi-2D Correlated Insulator FePS 3}

The discovery of the high temperature cuprate superconductors has lead to enormous scrutiny of materials which exhibit reduced dimensionality and have electronic structures in which electron correlation plays a significant role. ${ }^{25,26} \mathrm{FePS}_{3}$, a prototypical member of the $\mathrm{MPX}_{3}$ transition-metal phosphorus trichalcogenide series of compounds, bears qualitative similarity to the cuprates in these 
respects. 27,28 Similar to the cuprates and other systems having correlated electronic structures, an important issue in the $\mathrm{MPX}_{3}$ compounds concerns the degree of spatial localization of the $3 d$ manifold. This in turn is determined by the relative importance of on-site correlation and exchange interactions, crystal field splittings, and hybridization with neighboring atoms. Simple versions of these models mask much of the underlying complexity associated with quenching and/or delocalization of atomic multiplets. We recently have completed a detailed resonant photoemission (RESPES) and ARP studys of FePS 3 to probe the degree of localization of the $3 d$ levels. 29

Not surprisingly, there exist paradigmatic models to explain the electronic properties of these compounds. These can be classified as either 'ionic' and 'covalent' (or hybridized), though the true situation surely lies intermediate between these extremes. Our results have located the middle ground in this issue. For example, we can fit the iron-derived valence band photoemission features in this compound using atomic multiplet theory modified by crystal field effects. The parameters needed to fit the spectra, however, imply substantially reduced Racah exchange and correlation integrals relative to more 'ionic' iron compounds. The RESPES results can similarly be fitted with an atomic model, but the parameters imply somewhat delocalized Fe 3d levels. Finally, we observe, in some of the Fe 3d-derived features, substantial energy dispersion as a function of electron momentum. For some of the features, the band width is smaller than the observed and predicted multiplet splittings, implying that a localized description might be appropriate. For others, however, these quantities are comparable so that neither hybridization nor correlation effects dominate. We have suggested useful ways to think about these compounds which will hopefully motivate more detailed calculations than have been done in the past.

\section{Surface Bands on Rh(111)}

As a follow-up on our recent studies of $\mathrm{Pt}(111), 30,31$ we have completed measurements of the surface electronic structure of Rh(111). 32 These were motivated in part by unusual electronic structures predicted for carbidic carbon adsorbed on $\mathrm{Rh}(111)$ and $\mathrm{Ru}(0001),{ }^{33}$ though we were unable to produce any carbidic carbon structures experimentally. Our results for $\mathrm{Rh}(111)$ follow fairly directly those of $\mathrm{Pt}(111)$, so long as the Fermi level is rigidly shifted downward by $\sim 1 \mathrm{eV}$ to account for the lower d-band occupation. A surprising and possibly interesting deviation from rigid band behavior occurs near the $K$ point of the SBZ, where several surface states exist.

\section{B. Initial Efforts at the ALS}

\section{Beamline Commissioning}

The Advanced Light Source was officially commissioned in October, 1993. We are members of a 'Participating Research Team' (PRT) associated with undulator beamline 7.0 at the ALS. This beamline was commissioned for experiments the following winter and spring of 1994 . We have had about a year to overcome some of the unavoidable initial problems and to try to accomlpish some experiments. The 
postdoc currently supported by this grant (Eli Rotenberg) has been stationed at the ALS since the facility was commissioned. Along with Jonathon Denlinger, also supported by a DOE grant to Brian Tonner at the University of Wisconsin, he has been one of the two postdocs primarily responsible for commissioning the beamline and core level photoemission system. Happily, the two of them have made rapid progress in these endeavors and the future is indeed very bright.

Since we are active members of the PRT with personnel on site, we tend to get involved in three generic types of experiments. Firstly, we have some ideas of our own to pursue more or less independently of anyone else in the PRT. The time for these has been and continues to be very scarce since many groups are involved and the demand for time is high. The bulk of our activities in the past year have involved the other two kinds of experiments. There are firstly those in which we are 'major collaborators', meaning we contribute with other groups to all phases of the experiment, from conception through analysis and publication. Finally, there are experiments in which we play a more supporting role to help other groups with their experiments. This last type of experiment can be very enjoyable and can even take more time and effort on our part than the first two. Before describing a few initial results from the first two categories, we briefly review those from the third:

- We have helped install a soft $\mathbf{x}$-ray emission spectrograph belonging to Joseph Nordgren from Uppsala University in Sweden, and have participated in a small number of the many experiments his group has accomlished in the past year.

- We have collaborated with Brian Tonner's research group to collect resonant photoemission spectra from thin films of $\mathrm{C}_{60}$ and polystyrene. These are among the first RESPES studies above the $\mathrm{C} \mathrm{K}$ edge, and have lead to a new model for the relaxation processes near a core hole in these systems.

- We have collaborated with Norm Edelstein and David Shuh of LBL to measure the core level photoemission spectra from nano-Curie quantities for transuranic elements. This was an amusing experiment, since safety personnel outnumbered researchers by a significant margin. We got some good press for our efforts - it was written up in the DOE's ER Newsletter a few months back. A proposed application in verification of compliance to non-proliferation treaties seems far in the future.

\section{Core Level Photoemission and XPD}

The first functional end station on beamline 7.0 was optimized to perform core level photoemission. Most of our accomplishments in the first year have been in this area, and essentially all of these have been in collaboration with Brian Tonner and his postdoc Jonathan Denlinger. This system is able to accumulate a photoemission spectrum with excellent statistics and resolution in typically less than a minute. The apparatus has been mechanized to an extent that a set of complete, normalized spectra can be accumulated over a large range of emission angles under computer control. These capabilities provide 
one of the favored methodologies for accumulating x-ray photoelectron diffraction (XPD) data and, related to this, something that has come to be called a photoelectron hologram. Photoelectron diffraction is now a mature field. 34-36 Indeed, the P.I., in his PhD thesis, laid some of the groundwork for this technique, although he has not practiced it much over the intervening period. As its name implies, XPD invloves using the interference between the directly photoemitted and substrate back-scattered emission paths to probe surface geometric structures. If a large enough data set is available, then these diffraction patterns become spherical holograms that can be directly inverted to provide a $3 \mathrm{D}$ image in the vicinity of the emitting atom.

Over the past year, we have been involved in four separate XPD experiments:

- As a tune-up, we have accumulated a large '3D' data set - a large array of angles collected at many photon energies - on clean $\mathrm{Cu}(001)$. This can be inverted using standard techniques to yield the known structure of copper.

- One of the real strengths of XPD over most other surface structural techniques is simultaneous access to chemical and structural information. This is done by utilizing high energy resolution so that chemically shifted core levels can be resolved and their XPD patterns or holograms measured independently. The first such experiments we have undertaken have been on $\mathrm{Si}(111)-(7 \times 7)$ and Si(001) - (2x1). Both of these structures are fairly well understood, so this can also be considered a tune-up experiment to a degree. However, there is substantial discord in existing assignments of the observed shifted core levels to particular surface atoms in the reconstruction. There is thus significant interest in these experiments since the the origin of surface core level shifts and their relation to structure remains an active field, particularly on semiconductor surfaces.

- We have a partial XPD data set for the surface core levels on W(011). Again, our experiment is a test of a fairly well-known structure, though it is by no means a trivial effort given the reactivity of this surface. After we have completed the clean surface, we will undertake the hydrogen-covered surface which has been shown to undergo a (1x1) reconstruction in which the surface layer shifts laterally across the second layer. 37

- Finally, we have started and nearly completed an experiment on ultrathin manganese films on $\mathrm{Cu}(001)$. This prorotypical thin film magnetic metal system has an unknown structure and provides an opportunity to correlate magnetic and geometric structures.

\section{Coherent Soft X-ray Scattering}

Substantial interest exists in using the high brightness of second and third generation synchrotron radiation facilities to produce and use spatially coherent $x$-ray and soft $x$-ray beams for a number of applications. One of the P.I.'s primary motivations for moving to the ALS was the possibility 
of being involved in the qualitatively new science offered by this possibility. The particular research direction we have chosen to pursue in this regard is the scattering of coherent soft $x$-rays off fluctuating systems. Our motivations for pursuing such experiments will be discussed in more detail in the accompanying renewal proposal. Here, we will describe our first efforts to overcome the technical obstacles involved.

A spatially coherent light beam is one with wavefronts that are well-defined transverse to the propagation direction. This is generally the case in light emitted by well-designed lasers, but reasonably intense spatially coherent $x$-ray beams have only recently become avaliable. The high brightness of the ALS will provide substantially more 'coherent soft $x$-ray power' - that is - more photons in a spatially coherent beam - than has been available elsewhere. There are (at least) two ways to produce a spatially coherent beam on an undulator beamline at the ALS. Both rely fundamentally upon the fact the spatial coherence length of a light beam increases upon propagation. The mathematical statement of this is the van Cittert - Zernike theorem from optics, which relates the mutual coherence function of a partially coherent beam to the far-field diffraction pattern of a similarly-defined coherent beam. The two ways to produce spatially coherent soft $x$-rays at the ALS are:

- Up to about $500 \mathrm{eV}$ photon energy, the light emitted in the central cone by the $5 \mathrm{~cm}$ undulator on beamline 7.0 is spatially coherent due to the small emittance of the electron beam inside the undulator. Simply by placing a small (water-cooled) aperture in the undulator beam, one automatically gets spatially coherent light. At present, the U7.0 undulator beamline does not provide access to the raw undulator beam. However, a small group of researchers, of which we are members, has been assembled by Malcolm Howells of the Center for X-ray Optics at LBL to establish this capability within the next 6-12 months. This alternative will provide the highest possible flux and excellent spatial coherence with the disadvantage of having only fair temporal coherence $(\lambda \Delta \lambda \sim$ 100). In many applications, the temporal coherence is not particularly important and this addition to the beamline will be very useful to us.

- In principle, the limited temporal coherence of the direct undulator beam can be improved with a 'coherence length stretcher', i.e., a monochromator, without adversely impacting the spatial coherence properties. Indeed, a monochromator is installed on the U7 undulator and is used in essentially all other experiments. Unfortunately, the use of grazing incidence optics in a soft $x$-ray beamline implies figure errors and short-scale roughness in the optical surfaces that leaves the exit beam with ill-defined spatial coherence properties. The common technique for solving this problem is to insert two small apertures after the exit slit of the monochromator, one near the refocus point and another slightly downstream which is sized to accept the coherent fraction of the beam according to the van Cittert - Zernike theorem. 
In either of these two cases, the coherent power scales like the square of the wavelength, and the expected flux of coherent light is $\sim 10^{13}$ and $\sim 10^{9}$ photons/sec in the two cases above for photon energies near the carbon edge (280 eV). In December 1994, in collaboration with Larry Sorensen and Allen Price from the University of Washington, we attempted an experiment along the lines of the second design and achieved a coherent flux within an order of magnitude of the expected level. The power spectrum of the coherent light was extensively tested and was found to be adequate to measure temporal fluctuations on a frequency scale of $1 \mathrm{~Hz}-1 \mathrm{Mhz}$. This actually is a substantial and important result. Considering the mechanical and electronic instabilities that can be present along a 30 meter beamline, by the time one directs the beam exiting the monochromator through two $10-20$ micron apertures separated by $300 \mathrm{~mm}$ to separate the spatially coherent fraction, it is not obvious that the noise spectrum will have low enough amplitude to allow useful measurements of 'real' fluctuating signals. We showed that it does, but a substantial number of problems precluded any real experiments using this coherent light in our December run. We intend to pursue experiments along these lines again in the near future.

\section{Low Angle Diffraction from Nanostructures}

An increasing fraction of the work on this project involves scattering of $x$-rays and soft $x$-rays from thin films and nanostructured materials. This reflects our movement away from studies of the electronic structure of nominally clean surfaces and toward synthesis and analysis of exotic materials, as described more fully in the following renewal proposal. For this reason, in the fall of 1993, we wrote and were awarded a DOE University Research Instrumentation proposal to purchase and modify a new diffractometer. This will be utilized to a substantial degree to supplement our ALS activities. Since the URI program provides exclusively funds for instrumentation, the graduate student supported by this grant is involved in modifying and commissioning this apparatus. We describe this activity briefly here, while a more complete discussion of the science we will pursue is left for the renewal proposal.

The diffractometer purchased is a $\theta-\theta$ diffractometer with sufficient precision that low-angle diffraction from layered materials and thin films with d-spacings in the 10-10000 $\AA$ range can be measured. The modifications involve 1 ) adding analyzing crystals to both arms of the diffractometer so that high resolution can be achieved, 2) allowing for automated sample alignment with adequate precision for meaningful low-angle studies, and 3) addition of a high vacuum environmental chamber so that low and moderately high temperature studies can be undertaken in situ. Surprisingly, such an apparatus is not commercially available despite several obvious applications in thin film technology. Several companies have communicated substantial interest in such an apparatus. This had made the manufacturer (Scintag, Inc.) keenly interested in these modifications, and we are actively collaborating with them on this project. At present, all parts except the vacuum chamber have been completed and are being installed. 
D. References

1. R.H. Gaylord, K. Jeong, and S.D. Kevan, Phys. Rev. Lett. 62, 203 (1989).

2. K. Jeong, R.H. Gaylord, and S.D. Kevan, Phys. Rev. B 39, 2973 (1989).

3. E. Hulpke and J. Lüdecke, Phys. Rev. Lett. 68, 2846 (1992).

4. B. Kohler, P. Ruggerone, S. Wilke, and M. Scheffler, submitted to Phys. Rev. Lett. (1994).

5. T.B. Grimley, Proc. Roy. Soc. London 90, 751 (1967).

6. T.L. Einstein and J.R. Schrieffer, Phys. Rev. B 7, 3629 (1973).

7. T.L. Einstein, in Chemistry and Physics of Solid Surfaces, R. Vanselow,ed. (CRC, Boca Raton, 1979).

8. T.L. Einstein, Langmuir 7, 2520 (1991).

9. K.H. Lau and W. Kohn, Surface Sci. 75, 69 (1978).

10. N.H. March, Prog. Surface Sci. 25, 229 (1987).

11. J. Muscat, Prog. Surface Sci. 25, 211 (1987).

12. P. Johansson, Solid State Commun. 31, 591 (1979).

13. A.C. Redfield and A. Zangwill, Phys. Rev. B 46, 4289 (1992).

14. A.M. Bradshaw and M. Scheffler, J. Vac. Sci. Techol. 16, 447 (1979).

15. P.J. Feibelman, Ann. Rev. Phys. Chem. 40, 261 (1989).

16. J. Friedel, Adv. Phys. 3, 446 (1954).

17. S. Dhar, K.E. Smith, and S.D. Kevan, Phys. Rev. Lett. 64, 1448 (1994).

18. R.E. Peierls, Quantum Theory of Solids (Clarendon, Oxford, 1964).

19. K. Grzelakowski, I. Lyuksyutov, and E. Bauer, Surface Sci. 216, 472 (1989).

20. H.P. Bonzel, A.M. Bradshaw, and G. Ertl. Physics and Chemistry of Alkali Metal Adorption (Elsevier, Amsterdam, 1989).

21. D.M. Riffe, G.K. Wertheim, and P.H. Citrin, Phys. Rev. Lett. 64, 571 (1990).

22. E. Kneedler, A.B. Andrews, W.-K. Choi, and S.D. Kevan, Phys. Rev. B 51, 1823 (1995).

23. E. Kneedler, D. Skelton, K.E. Smith, and S.D. Kevan, Phys. Rev. Lett. 64, 3151 (1990).

24. E. Kneedler, K. Smith, and S.D. Kevan, Phys. Rev. B 44, 8233 (1991).

25. H. Ehrenreich and D. Turnbull. Solid State Physics (Academic, Boston, 1989).

26. P. Fulde, Electron Correlations in Molecules and Solids (Springer, Berlin, 1991).

27. W. Klingen, R. Ott, and H. Hahn, Z. Anorg. Allg. Chem. 396, 271 (1973).

28. W. Klingen, G. Eulenberger, and H. Hahn, Z. Anorg. Allg. Chem. 401, 97 (1973).

29. W.-K. Choi, E. Kneedler, A.B. Andrews, and S.D. Kevan, Phys. Rev. B 50, 15276 (1994).

30. W. Di, K.E. Smith, and S.D. Kevan, Phys. Rev. B 43, 12062 (1991).

31. W. Di, K.E. Smith, and S.D. Kevan, Phys. Rev. B 45, 3652 (1992).

32. W. Di and S.D. Kevan, Phys. Rev. B 49, 4821 (1994).

33. P.J. Feibelman, Phys. Rev. B 26, 5347 (1982).

34. W.F. Egelhoff, Crit. Rev. Solid State and Mat. Sci. 16, 213 (1990).

35. C.S. Fadley, in Synchrotron Radiation Research: Advances in Surface Science, R.Z. Bachrach, eds. (Plenum, New York, 1990).

36. D.P. Woodruff, in Angle-Resolved Photoemission, S.D. Kevan, eds. (Elsevier, Amsterdam, 1992).

37. J.W. Chung, S.C. Ying, and P.J. Estrup, Phys. Rev. Lett. 56, 749 (1986). 


\section{E. Personnel}

Several graduate students have been supported on this project over the past three years. Two of the three students who were supported for work at the NSLS, Di Wei and Rick Kneedler, finished and defended their Ph.D. theses and graduated in the summer and fall of 1993, respectively. Kneedler is an NRC Postdoctoral associate at the Naval Research Laboratory, and Wei is a postdoctoral associate at the University of Sherbrooke in Canada. The third student, Sanjay Dhar, is completing his thesis and will soon take a job at Applied Magnetics, a small company in Santa Barbara, California. Two new students are now working on this project. Abed Khooli is in charge of construction and commissioning our new diffractometer. His thesis work will focus on diffraction from synthetic multilayers or diffraction or coherent scattering from polymeric films. A second student, Kristen McCutcheon, is partially supported by a Department of Education fellowship, though some of her her research activities at synchrotron radiation facilities are supported by this grant. Her focus is upon diffusion and nucleation at buried interfaces.

From its inception, the project has also supported a postdoctoral associate who has always been stationed at either the NSLS or the ALS. The final NSLS postdoc, Ballard Andrews, left for a position at the NSLS but supported by Los Alamos National Laboratory in the spring of 1993. In the fall of 1994, a new postdoc, Eli Rotenberg, was hired and stationed at the ALS. He continues in this position at present. 
F. Publications from Sponsored Research

1. "Angle-resolved Photoemission Study of the Clean and Hydrogen-Covered Pt(111) Surface", Wei Di, K.E. Smith, and S.D. Kevan, Phys. Rev. B 45, 3652 (1992).

2. "A Normal Incidence VUV Emission Spectrometer", Z. Tang, Z. Xu, and S.D. Kevan, Rev. Sci. Inst. 63, 1896 (1992).

3. "Photoemission Study of the Surface Electronic Structure on Mo(001) and Mo(001)-2H", K.E. Smith and S.D. Kevan, Phys. Rev. B 45, 13642 (1992).

4. "Soft X-ray Emission and Inelastic Scattering at the C K-edge of Diamond", Y. Ma, N. Wassdahl, P. Skytt, J. Guo, J. Nordgren, J.-E. Rubensson, T. Boske, W. Eberhardt, and S.D. Kevan, Phys. Rev. Lett 69, 2598 (1992).

5. "Determination of Atomic Density Profiles in Synthetic Multilayers by Anomalous X-ray Diffraction", Z. Tang, Z. Xu, S.D. Kevan, T. Novet, and D.C. Johnson, Appl. Phys. Lett. 62, 1771 (1993).

6. "The Effect of Structural Incoherence on the Low Angle Diffraction Pattern of Synthetic Multilayer Materiais", Z. Xu, Z. Tang, S.D. Kevan, T. Novet, and D.C. Johnson, J. Appl. Phys. 74, 905 (1993).

7. "The Use of Superlattices to Probe and Control Reactions at Buried Interfaces", L. Fister, T. Novet, Z. Xu, S.D. Kevan, and D.C. Johnson, Mat. Sci. and Eng. A 162, 115 (1993).

8. "Distinguishing between Coherent Interdiffusion and Incoherent Roughness in Synthetic Multilayers using X-ray Diffraction", Z. Xu, Z. Tang, S.D. Kevan, T. Novet, and D.C. Johnson, Mat. Res. Soc. Proc. 280, XXXX (1993).

9. "Electronic Structures of $\alpha-\mathrm{Fe}_{2} \mathrm{O}_{3}$ and $\mathrm{Fe}_{3} \mathrm{O}_{4}$ from $\mathrm{O}$ K-edge Absorption and Emission Spectroscopy", Y. Ma, P.D. Johnson, N. Wassdahl, J. Guo, P. Skytt, J. Nordgren, S.D. Kevan, J.E. Rubensson, T. Böske, and W. Eberhardt, Phys. Rev. B 48, 2109 (1993).

10. "Surface Electronic Structure of Rh(111)", Wei Di and S.D. Kevan, Phys. Rev. B 49, 4821 (1994).

11. Fermi Contours and Surface Dynamical Phenomena", S. D. Kevan, Surface Sci. 307,832 (1994).

12. "Delocalization of the Fe $3 d$ Levels in the Quasi-2D Correlated Insulator FePS 3 ", Won-Kook Choi, E. Kneedler, and S.D. Kevan, Phys. Rev. B 50, 15276 (1994).

13. "Fermi Contours and Adsorbate Periodicities: $\mathrm{O} / \mathrm{Mo}(011)$ and $\mathrm{O} / \mathrm{W}(011)^{n}$, S. Dhar, K.E. Smith, and S.D. Kevan, Phys. Rev. Lett. 73, 1448 (1994).

14. "Alkali Chemisorption on Ta(011)", E. Kneedler, A.B. Andrews, and W.-K. Choi, and S.D. Kevan, Phys. Rev. B. 51, 1823 (1995).

15. "Low Angle $X$-ray Diffraction as a Probe of Reactions at Buried Interfaces and as a Characterization Technique for Thin Films", T. Novet, S.D. Kevan, and D.C. Johnson, Mat. Sci. and Eng. XXX, XXXX (1995).

16. "First Results from the Spectromicroscopy Beamline at the Advanced Light Source", J.D. Denlinger, E. Rotenberg, T. Warwick, G. Visser, J. Nordgren, J.H. Guo, P. Skytt, S.D. Kevan, K.S. McCutcheon, D. Shuh, J. Bucher, N. Edelstein, J.G. Tobin, and B.P. Tonner, Rev. Sci. Inst. 66, XXXX (1995).

17. "Surface Core Level Photoelectron Diffraction of Surface Reconstructions", E. Rotenberg, J. Denlinger, S.D. Kevan, and B.P. Tonner, Mat. Res. Soc. Proc. XXX, XXXX (1995).

18. "Surface States on Metals", S.D. Kevan, book chapter in Electronic Structure of Surfaces, K. Horn and M. Scheffler, eds. (Elsevier, Amsterdam, 1995). 


\section{G. Presentations on Sponsored Research}

1. "Determination of Atomic Density Profiles in Synthetic Multilayers by Anomalous X-ray Diffraction", American Vacuum Society Meeting, Chicago, November, 1992.

2. "ARP Study of Clean and H-Covered Rh(111)", American Physical Society Meeting, Seattle, March, 1993, talk delivered by Di Wei.

3. "X-ray Absorption as a Probe of Binary Multilayers", American Physical Society Meeting, Seattle, March, 1993, talk delivered by K. McCutcheon.

4. "Alkali Chemisorption on Ta(011)", American Physical Society Meeting, Seattle, March, 1993, talk delivered by $\mathrm{R}$. Kneedler.

5. "Fermi Contours and Surface Dynamical Phenomena", invited talk at a Symposium on AngleResolved Photoemission, Ringsberg, Germany, May, 1993.

6. "Fermi Contours and Surface Dynamical Phenomena", invited talk at the 13th European Conference on Surface Science, Warwick, UK, September 1993.

7. "Alkali Chemisorption on Ta(011)", Physical Electronic Conference, June, 1993, Rennselaer Polytechnic Institute, Troy, N.Y., talk delivered by R. Kneedler.

8. "Alkali Chemisorption on Ta(011)", Topical Conference on Alkali Adsorption, Penn State University, June, 1993, talk delivered by R. Kneedler.

9. "Are Surface States Important?", invited talk at a WE-Heraues Seminar on Electronic Surface States and Interface States on Metallic Systems, Bad Honnef, Germany, October, 1994.

10. "Are Surface States Important?", U of O Condensed Matter Physics Seminar delivered by S. Kevan, October, 1994.

11. "First Results from the Spectromicroscopy Beamline at the Advanced Light Source", Synchrotron Radiation Instrumentation 1994, talk delivered by J.D. Denlinger.

12. "Surface Core Level Photoelectron Diffraction of Surface Reconstructions", Annual Meeting of the Materials Research Society, Boston, November, 1994, talk delivered by E. Rotenberg.

13. "Core Level Photoelectron Diffraction Studies of Silicon Surface Reconstructions", March Meeting of the American Physical Society, March, 1994, San Jose, talk delivered by E. Rotenberg.

14. "Synchrotron Photoelectron Spectroscopy Studies of Actinides", March Meeting of the American Physical Society, March, 1994, San Jose, talk delivered by J. Denlinger. 